\title{
Unbiased antimicrobial resistance detection from clinical bacterial isolates using proteomics
}

\author{
Christian Blumenscheit ${ }^{1}$, Yvonne Pfeifer ${ }^{2}$, Guido Werner², Charlyn John', Andy \\ Schneider ${ }^{1}$, Peter Lasch $^{1}$ and Joerg Doellinger ${ }^{1}$ \\ ${ }^{1}$ Robert Koch Institute, Centre for Biological Threats and Special Pathogens, Proteomics and \\ Spectroscopy (ZBS6), Berlin, Germany \\ ${ }^{2}$ Robert Koch Institute, Nosocomial Pathogens and Antibiotic Resistance (FG13), \\ Wernigerode, Germany
}

Correspondence: Joerg Doellinger (Email: doellingerj@ rki.de, phone: +49 30-18754 2373)

Running Title: Unbiased antimicrobial resistance detection using proteomics

Keywords: antibiotic resistance mechanism/AMR/LC-MS/proteomics/mass spectrometry/bacteria/dataindependent acquisition/carbapenemases/ESBL/mcr 


\section{Abbreviations}

AGC: Automatic gain control

AMR: Antimicrobial resistance

ARO: Antibiotic Resistance Ontology

AST: Antimicrobial Susceptibility Testing

BLDB: Beta-lactamase database

BMD: Broth microdilution

CAA: 2-chloroacetamide

CARD: Comprehensive Antibiotic Resistance Database

DB: Database

DDA: Data dependent acquisition

DIA: Data independent acquisition

ESBL: Extended spectrum beta lactamases

ESKAPE: Enterococcus feacium, Staphylococcus aureus, Klebsiella pneumoniae,

Acinetobacter baumannii, Pseudomonas aeruginosa, Enterobacter spp.

fa-SPEED: filter-aided Sample Preparation by Easy Extraction and Digestion

MALDI-TOF: Matrix-assisted laser desorption/ionization Time-of-flight

MS: Mass spectrometry

MSA: Multiple sequence alignment

NCE: Normalized collision energy

NGS: Next generation sequencing

PBS: Phosphate-Buffered Saline

PCR: Polymerase chain reaction

PRM: Parallel Reaction Monitoring

PTFE: Polytetrafluoroethylene

RKI: Robert Koch Institute

SPEED: Sample Preparation by Easy Extraction and Digestion

TCEP: tris(2-carboxyethyl)phosphine

TFA: Trifluoroacetic acid

WGS: Whole genome sequencing 


\begin{abstract}
$\underline{\text { Abstract }}$
Antimicrobial resistance (AMR) poses an increasing challenge for therapy and clinical management of bacterial infections. Currently, antimicrobial resistance detection often relies on phenotypic assays, which are performed independently from species identification. Although genomics-based approaches are increasingly being proposed as possible alternatives for resistance detection, the analysis of proteins should be superior to gene or transcript sequencing when it comes to phenotype prediction from molecular data as the actual resistance against antibiotics is almost exclusively mediated by proteins. In this study, we present a unbiased proteomics workflow for detecting both, bacterial species and AMR related proteins in the absence of secondary antibiotic cultivation in less than $4 \mathrm{~h}$ from a primary culture. The method was validated using a sample cohort of 7 bacterial species and 11 AMR determinants represented by 13 protein isoforms which resulted in a sensitivity of $98 \%$ (100\% with vancomycin inference) and a specificity of $100 \%$ with respect to AMR determinants. This proof-of concept study suggests a high application potential of untargeted proteomics in clinical microbiology.
\end{abstract}




\section{$\underline{\text { Introduction }}$}

The rapid emergence and spread of antimicrobial resistant bacteria is a major threat for global health (WHO, 2014). Therefore, the rational use of antibiotics is of utmost importance, which in turn depends on fast and accurate detection of clinically relevant resistances (O`Neill, 2016; WHO, 2015). The most important results in guiding antimicrobial therapy are a correct species identification and a reliable antimicrobial susceptibility testing (AST). Currently no method is applied for routine diagnostics of patient samples, which addresses both needs simultaneously. The last decade has witnessed a revolution in routine clinical microbial diagnostics. MALDI-ToF MS enables today rapid, accurate and cost-effective biotyping at high sample throughput (Patel, 2015; van Veen et al, 2010). On the contrary, the determination of antibiotic resistance relies mainly on the analysis of bacterial growth in the presence of antibiotics. This phenotypic approach is often complemented by molecular analysis of targeted genes using polymerase chain reaction (PCR). Due to the high relevance of antimicrobial resistance detection, many different methods are currently under development with the aim at reducing the time-to-results and improving the accuracy (van Belkum et al, 2020; van Belkum \& Dunne, 2013). These methods split into two main categories. The first approach aims to improve phenotypic methods by increasing the sensitivity of detecting bacterial growth at different conditions and so reduce time to results (Dubourg et al, 2018). The second approach uses molecular analysis methods to predict antibiotic resistance from molecular signatures and is currently dominated by next generation sequencing (NGS) (Su et al, 2019). Molecular analysis using 'omics'-technologies offers the potential for accurate prediction of every known resistance phenotype without the need for parallel cultivation in the presence of different antibiotics and without its inherent limitations in transferring the results from the specialized lab cultures into the clinics. In theory, the analysis of proteins should be superior to gene or transcript sequencing when it comes to phenotype prediction as resistances are almost exclusively mediated by proteins (Alcock et al, 2020). Therefore, mass spectrometry-based proteomics is a promising candidate as a "one-for-all" solution for addressing the above mentioned needs in clinical microbiology (Welker \& van Belkum, 2019). However, several limitations have hampered the breakthrough of this technology so far. Direct detection of the actual proteins conferring resistance by whole cell mass spectrometry biotyping using MALDI-ToF MS is hard to imagine even in the further future (Welker \& van Belkum, 2019). Therefore MALDI-ToF MS is currently evaluated as a readout technique for phenotypic antimicrobial susceptibility testing (AST) (Correa-Martínez et al, 2019; Lange et al, 2014; Nix et al, 2020; Sparbier et al, 2016). In contrast, liquid chromatography coupled to tandem-mass spectrometry (LCMS/MS) offers the potential for molecular detection of all known antimicrobial resistance determinants in combination with bacterial species identification. However, no such unbiased LC-MS/MS workflow exists so far. Instead, the majority of AMR-related proteomics studies rely on targeted analysis of selected peptides representing AMR determinants (Cecchini et al, 2018; Charretier et al, 2015; Foudraine et al, 2019; Hassing 
et al, 2016; Strich et al, 2019). The results of this studies are quite promising, as they prove that sensitive and specific detection at least of some AMR phenotypes based on proteins is possible. Untargeted proteomics on the other hand has not been evaluated systematically for unbiased antimicrobial resistance prediction most probably due to major technical limitations (Chen et al, 2019).

In this study, we present an untargeted LC-MS/MS-based proteomics workflow, which was developed to address the special requirements of antimicrobial resistance detection in clinical microbiology. The proposed workflow not only predicts AMR phenotypes from clinical isolates in an unbiased manner with high sensitivity and specificity using large-scale sequence databases but allows also to simultaneously identify the bacterial species.

\section{$\underline{\text { Results }}$}

General workflow. In this study, a proteomic workflow is presented, which enables bacterial species identification and antimicrobial resistance detection (See Figure 1 for an illustration) simultaneously. The method involves preparation of bacterial isolates cultivated in the absence of antibiotics using the filteraided SPEED protocol (fa-SPEED)(Doellinger et al, 2020b). This method is rapid ( $30 \mathrm{~min}$ ), convenient, automatable, and even more important universal for all types of microorganisms, including spore-forming Gram-positive bacteria. The LC-MS/MS measurement relies on data-independent acquisition (DIA), which circumvents the protein detection bias caused by the stochastic nature of data-dependent acquisition (DDA). This strategy allows collection of proteomes with deep coverage (Doellinger et al, 2020a). However, it introduces big challenges for data analysis, which need to be covered by tailor-made concepts. In our workflow the peptide identification is based on in silico spectral library prediction and neural network supported data analysis using DIA-NN (Demichev et al, 2019). However, this approach cannot be used to identify bacteria from very large sequence databases, such as all bacterial entries of the UniprotKnowledgebase (O. n. s. o. r. t. i. u. m. UniProt, 2019). Therefore, we utilized a LC-MS ${ }^{1}$ based method for microbial species identification, which has been adopted from MALDI-ToF MS biotyping and relies on $\mathrm{LC}_{\mathrm{MS}}{ }^{1}$ pattern analysis (Lasch et al, 2020). The proteome of the identified species is then employed for predicting a sample-specific spectral library, which are in turn used as a background for peptide identification from the Comprehensive Antibiotic Resistance Database (CARD) (Alcock et al., 2020). The results are interpreted by means of an in-house R script, named rawDIAtect, which reports the AMR phenotype. The performance of the proposed workflow was evaluated using a panel of 29 bacterial isolates covering 7 species and 11 different AMR determinants represented by 13 protein isoforms, including multidrug-resistant clinical isolates and trans-conjugated bacteria (Table 1). 
Species identification based on LC-MS ${ }^{1}$ biotyping. The LC-MS biotyping approach enables simple and rapid bacterial identification at the species level from very large sequence databases. The method relies on $\mathrm{MS}^{1}$ data and thus can be simply integrated into the analysis workflow of DIA data. The results of the LC$\mathrm{MS}^{1}$ biotyping are summarized in Table 2.

Out of the 29 samples tested 27 were correctly identified at the species level. Only two E. coli samples (transconjugants 443-16_K1 and 371-12_K1) were misclassified as Shigella boydii and Shigella flexneri, respectively. Shigella species are closely related to Escherichia coli and some methods, including MALDIToF MS, cannot be used for reliable differentiation of both species (Khot \& Fisher, 2013; Paauw et al, 2015), which is a well-known issue in microbiology. Furthermore, the serovar of the Salmonella enterica isolate 50-07 was misclassified. The use of peptide mass profiles from LC-MS ${ }^{1}$ data for species identification is a quite new concept, which has an identification rate of $93 \%$ within this dataset. This is well within the range of reported values for MALDI-ToF biotyping, which is a matured and already legally approved technique (Hou et al, 2019). Presumably, further improvements of the data analysis workflows for LC-MS ${ }^{1}$-based biotyping should enable even higher identification rates in the future, e.g. considering the fact, that no well-curated database for this purpose is currently available.

Sensitivity of AMR detection by proteomics. AMR detection by proteomics relies on the assumption that the resistance determinants are expressed even in the absence of antibiotics. We hypothesized, that the detection of proteins conferring resistance is mainly a matter of sensitivity. The proposed experimental workflow provides sufficient analytical sensitivity to cover nearly complete proteomes of clinical isolates. This hypothesis was tested for a panel of 30 samples (29 isolates in 30 measurements, double E. coli negative control), which were characterized previously using phenotypic AST, PCR and Sanger sequencing (Table $1)$.

AMR detection by proteomics was based on the unmodified whole CARD (Full CARD, see above) without filtering short sequences or redundancies. Figure 2 compares outcomes of LC-MS/MS-based AMR-related protein family detection (y-axis) with the corresponding PCR/Sanger reference data (x-axis). The correctness of MS identifications is color coded as either verified by PCR (green), additional (dark orange) or missing (purple). The number of unique peptides of each AMR protein family is denoted in each tile.

In total, 43 out of 45 expected proteins conferring resistance were detected by MS. Only VanB ligase was missing in one E. faecium samples (UW16517). However, in this sample vancomycin resistance was confirmed by detection of both parts of the two-component system $\operatorname{VanS}_{\mathrm{B}}$ and $\mathrm{VanR}_{\mathrm{B}}$ (Figure 2). Furthermore, drug classes associated with protein isoforms were compared with the phenotypic data (Table S 1). The resulting sensitivity was $96 \%$ considering samples, $91 \%$ considering drug classes and $98 \%$ with respect to AMR protein families using a strict matching to genes identified by PCR. When the presence of the VanB protein in the E. faecium samples UW16517 is inferred from the expression of its regulatory 
system $\operatorname{VanS}_{\mathrm{B}} / \mathrm{VanR}_{\mathrm{B}}$, the sensitivity is $100 \%$ at all levels (Figure 3). We also observed 21 additional protein hits, which were not detected by PCR/sanger sequencing (Figure 2). Among the additional hits, AAC(6)-Ii was found in all E. faecium isolates. The respective resistance gene is known to be intrinsic in this species and was therefore not tested by PCR. (Galimand et al, 2011; Hollenbeck \& Rice, 2012). Due to the nature of the PCR detection being a targeted method, which is not able to cover the heterogeneity of all AMR proteins, this discrepancy in comparison to the proteomics results is expectable. The results of the AMRrelated protein identifications including their isoform information are summarized in supplementary Table S 2.

Specificity of AMR detection by proteomics. The specificity of the proteomic approach was analyzed after reduction of the CARD to contain only the potential PCR targets (Figure 4, Whitelisted CARD approach). In contrast to Figure 2, we compared in the example of Figure 4 the MS hits not only against the PCR results, but also to NGS data (Table S 2) in order to compensate for the limitations of the PCR. The results of 30 LC-MS measurements on the y-axis are matched against the possible PCR and NGS hits on the $\mathrm{x}$-axis Again, 43 out of 45 expected proteins conferring resistance were identified, while VanB ligase was again missing in one E. faecium samples. The AAC(6)-Ib-like hits in all three negative controls of our E. feacium samples in the Full CARD approach were not detected anymore by the Whitelisted approach. Furthermore, no AMR determinants were detected in the E. coli negative controls. AAC(3)-like proteins were not tested in the Whitelisted CARD approach. The additional hits in both isolates 17/11 K1 and 33016-K1 identified by the Full CARD approach were also not detected anymore, indicating good agreement between PCR and LC-MS results if equal reference sequences are used. In total, no resistance-related proteins were identified in the 5 negative control samples, which resulted in a specificity of $100 \%$ considering samples, drug classes and AMR protein families (Figure 3). 


\section{$\underline{\text { Discussion }}$}

Accurate identification of the microbial species and correct determination of antimicrobial resistance pattern are of paramount importance to assure adequate treatment of patients suffering from bacterial infections. The aim of this proof-of-concept study was thus to establish an untargeted proteomics workflow suitable for the comprehensive characterization of pathogenic bacteria that includes both, species identification and AMR type determination. In contrast to culture-based phenotypic methods, this molecular proteomic-based analysis of clinical isolates has the potential for accurate prediction of every known resistance phenotype in a truly untargeted manner. Currently, genomics is the only 'omics'-technology which has been systematically evaluated for this purpose, although the analysis of proteins could offer some advantages due to its close relationship to the phenotype. Proteins directly mediate almost all resistant phenotypes and can be therefore considered ideal targets for antimicrobial susceptibility testing (AST). The potential of proteomics for AMR detection in general has been shown earlier using targeted analysis of distinct proteins. However, as antibiotic resistance databases, such as the Comprehensive Antibiotic Resistance Database (CARD), which currently includes > 1600 AMR protein families, are continuously growing and many of these gene families are highly heterogeneous a targeted approach for comprehensive AMR detection seems not effective (Alcock et al., 2020). So far major technological challenges have prevented untargeted proteomics from being a competitive alternative to NGS. In this study, we propose a proteomics workflow, which combines several recent technological developments into a workflow strategy, which was not possible until recently. The strategy is based on the in-silico prediction of sample-specific peptide spectral libraries as well as universal resistance-related peptide spectral libraries obtained by deep learning. This enables the use of DIA-based proteomics, which records the entity of peptide fragments in a sample above its sensitivity threshold in an unbiased manner with deep proteome coverage. The prediction of samplespecific background libraries for AMR detection is based on the results of a recently introduced strategy for biotyping bacteria using LC-MS ${ }^{1}$ data, whose recording can easily be integrated into DIA measurements (Lasch et al., 2020). This approach resulted in an identification accuracy of $93 \%$ with a runtime of $\sim 1 \mathrm{~min}$ on a decent desktop PC using a database with 8540 bacterial entries. The workflow further involves very rapid ( 30 $\mathrm{min})$ and universal sample preparation using acid extraction, without the need for protocol adaptation to certain bacterial species. Potentially, this method, termed SPEED, should enable to fully automate sample preparation including bacterial cell lysis in the future, as it uniquely omits the need for physical disruption (Doellinger et al., 2020a).

The performance of this workflow for AMR detection was tested with a panel consisting of 20 clinical isolates and 9 E. coli transconjugants containing a broad range of beta-lactamases (SHV-1/-11/-12, TEM1/-52, CMY-2, CTX-M-15/-9, KPC-2/-3, NDM-7, OXA-48/-1, VIM-1), the aminoglycoside acetyltransferases AAC(6)-like, $v a n A$ - and vanB-type vancomycin resistances and plasmid mediated colistin 
resistance (Mcr). The sensitivity was found to be $98 \%$ (100\% with vancomycin inference) and the specificity was $100 \%$, with respect to AMR-related protein families (cf. Figure 3). This demonstrates that antimicrobial resistance can be sensitively detected by protein analysis even in the absence of antibiotics during cultivation and demonstrates the high potential of untargeted proteomics for routine diagnostics in clinical microbiology.

The current workflow used in this proof-of-concept study takes $\sim 4$ h from bacteria to results, of which $3.3 \mathrm{~h}$ is needed for the actual LC-MS/MS measurement. Recent advancements in MS instrumentation in combination with liquid chromatography using higher flow rates have dramatically increased the proteome coverage when using short LC gradient lengths (<30 min) (Bache et al, 2018; Meier et al, 2018; Messner et al, 2020). Therefore, we expect, that the LC-MS/MS measurement time needed to provide sufficient depth for clinical microbiology should massively decrease in the future and thus increase sample throughput. Ultimately, this could end up in the unbiased characterization of bacteria directly from clinical specimen without any cultivation.

An important consideration for routine clinical applications of this workflow is the establishment of curated and validated peptide databases as the use of unprocessed publicly available protein sequence databases suffers from entries with low quality. Such peptide databases could also include information on discriminatory sequences, which could be used to distinguish clinically relevant protein isoform families, such as ESBL and non-ESBL variants of SHV and TEM, which is not possible with the current workflow. Antimicrobial resistance is often associated with a high genetic variability. However, the clinical relevance of the vast majority of genetic variants is unknown. RawDIAtect was able to discriminate between AMR protein subfamilies like carbapenemase producing OXA 48-like proteins and non-carbapenemase producing OXA 1-like proteins and further reports all possible isoforms for the detected peptide sequences as sequence coverage for most proteins is incomplete in many instances.

The output of rawDIAtect already contains information on the protein abundance. This data could potentially be used for the categorization of isolates into susceptible, intermediate or resistant strains just as the current use of minimum inhibitory concentration (MIC) breakpoints and so enable antimicrobial susceptibility testing (AST). A correlation between abundance of resistance determinants and MICs has already been demonstrated before for RNA \& NGS data (Bhattacharyya et al, 2019; Khaledi et al, 2016; Nguyen et al, 2019). These further improvements of the workflow could potentially turn this proof-of concept workflow into a method ready for routine applications in clinical microbiology. 


\section{$\underline{\text { Materials and Methods }}$}

Bacterial Cultivation. A panel of antimicrobial resistant bacteria consisting of 29 isolates with 7 species including Gram-negative (Escherichia coli $(\mathrm{n}=9)$, Klebsiella pneumoniae $(\mathrm{n}=7)$, Citrobacter freundii $(\mathrm{n}=1)$, Enterobacter hormaechei $(\mathrm{n}=1)$, Salmonella enterica ser. Infantis $(\mathrm{n}=1)$, Salmonella enterica ser. Typhimurium $(\mathrm{n}=1)$ ) and Gram-positive bacteria (Enterococcus faecium $(\mathrm{n}=9)$ ) was analyzed in $30 \mathrm{LC}$ MS/MS runs (double E. coli negative control) (Table 1). The Gram-negative isolates contained various $\beta$ lactamases including carbapenemases and extended-spectrum $\beta$-lactamases (ESBL). Some isolates additionally carried aminoglycoside acetyltransferases (AAC(6)-Ib-like) and plasmid-mediated colistin resistance (Mcr-1). The E. coli isolates were $\beta$-lactamase producing transconjugants that were obtained from clinical donor strains by mating experiments using the sodium azide-resistant recipient strain E. coli J53 $A z i^{r}$. The enterococci contained various vancomycin resistance gene clusters (vanA-type, vanB-type) including genes encoding a VanA or a VanB ligase, respectively. Cells were streaked under sterile conditions on Mueller Hinton II Agar (Becton Dickinson, Heidelberg, Germany) and aerobically grown at $37^{\circ} \mathrm{C}$ overnight for at least $15 \mathrm{~h}$ prior to proteomic sample preparation.

Antimicrobial susceptibility testing (AST). Antimicrobial susceptibilities of all 29 isolates were determined by the use of broth microdilution (BMD) and VITEK 2 card AST N248 according to DIN58940 and EUCAST guidelines v10.0 (www.eucast.org). In total, 19 antibiotics were tested, including ampicillin, piperacillin, piperacillin/tazobactam, cefotaxime, ceftazidime, cefoxitin, cefepime, meropenem, imipenem, gentamicin, amikacin, tobramycin, nalidixic acid, ciprofloxacin, moxifloxacin, fosfomycin, tigecycline, sulfamethoxazole/trimethoprim and colistin. Additionally, ETEST ${ }^{\circledR}$ with imipenem and ertapenem (bioMérieux, Nuertingen, Germany) were performed for selected isolates. AST of enterococcal isolates was determined with BMD and according to EUCAST standards and guidelines. For isolates that do not have a clinical breakpoint, corresponding ECOFF values were applied to discriminate the wildtype population from non-wildtype (= resistant).

PCR-based detection of resistance genes. The presence of various $\beta$-lactamase genes $\left(\right.$ bla $_{\mathrm{NDM}}$-like, $b l a_{\mathrm{VIM}}$ -

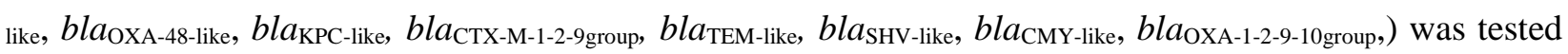
by PCR and subsequent Sanger sequencing using previously described primers and protocols (Gröbner $e t$ al, 2009; Pfeifer et al, 2011; Turton et al, 2006). Additionally, PCR screening and subsequent Sanger sequencing for genes contributing to resistance to aminoglycosides (aac(6')-Ib-like) and colistin ( $m c r-1$ ) was performed as described elsewhere (Liu et al, 2016; Park et al, 2006). The presence of vancomycin resistance gene clusters was determined by detecting corresponding ligase genes vanA and vanB by a multiplex PCR according to an accredited in house protocol (Werner et al, 2012). 
NGS-based detection of resistance genes. NGS data were analyzed in order to identify resistance genes and thus confirm PCR and LC-MS/MS results. Sequence data were already publicly available for the $K$. pneumoniae isolates (Becker et al, 2018). Both Salmonella isolates as well as the $C$. freundii and the $E$. hormaechei complex isolates were sequenced in the course of this study with Illumina HiSeq in a paired 150 bp manner with an aimed coverage of 20x by LGC Genomics (Berlin, Germany). The bacterial DNA was extracted using the DNeasy Blood and Tissue kit according to the protocol of the manufacturer (Qiagen, Hilden, Germany). Quantification was carried out using the Qubit dsDNA HS Assay Kit (Invitrogen/ Thermo Fisher Scientific, Karlsruhe, Germany). Library preparation with 1 ng of extracted DNA was done by applying the Nextera XT DNA Library Prep Kit according to the manufacturer's instructions (Illumina, San Diego, CA). Libraries were subjected to whole-genome sequencing using an Illumina ${ }^{\circledR}$ MiSeq benchtop devise (Illumina, San Diego, CA) in paired-end mode (v3 chemistry; 2x 300 bp). All reads were trimmed and filtered using fastp on default settings (https://github.com/OpenGene/fastp, v0.20.1) and assemblies were generated de-novo from trimmed and filtered reads by Unicycler using the short only read assembler option (https://github.com/rrwick/Unicycler, v0.4.8) without read correction and normal bridging. Detection of AMR relates genes was done with Abricate (https://github.com/tseemann/abricate, v1.0.1) using the CARD database and Kleborate (https://github.com/katholt/Kleborate, v1.0.0). The NGS results are summarized in Table S 2.

Sample preparation for proteomics. Bacteria were prepared for mass spectrometry by filter-aided Sample Preparation by Easy Extraction and Digestion (fa-SPEED) as described previously (Doellinger et al., 2020b). In brief, bacterial cells were harvested using an inoculating loop, washed twice with PBS and pelleted for $5 \mathrm{~min}$ at 5,000 x g. Pellets were resuspended in trifluoroacetic acid (TFA) (Optima® LC/MS, Thermo Fisher Scientific, Schwerte, Germany) (sample/TFA 1:4 (v/v) and incubated at $70^{\circ} \mathrm{C}$ for $3 \mathrm{~min}$. Samples were neutralized with $2 \mathrm{M}$ TrisBase using $10 \mathrm{x}$ volume of TFA and further incubated at $95^{\circ} \mathrm{C}$ for 5 min after adding Tris(2-carboxyethyl)phosphine (TCEP) to a final concentration of $10 \mathrm{mM}$ and 2chloroacetamide (CAA) to a final concentration of $40 \mathrm{mM}$. Protein concentrations were determined by turbidity measurements at $360 \mathrm{~nm}(1 \mathrm{AU}=0.67 \mu \mathrm{g} / \mu \mathrm{L})$ using the NanoPhotometer® NP80 (Implen, Westlake Village, California). Note, that the turbidity values are instrument dependent, for further details see Doellinger et. al 2020 (Doellinger et al., 2020b). $40 \mu \mathrm{g}$ proteins were mixed with acetone (Uvasol® for spectroscopy, Merck, Darmstadt, Germany) to obtain a final concentration of $80 \%$ acetone (v/v) and pipetted on an Ultrafree-MC PTFE membrane $0.2 \mu \mathrm{m}$ filter (Merck Millipore, Burlington, MA). The filter was incubated for $2 \mathrm{~min}$ at room temperature and was then centrifuged at $5000 \mathrm{xg}$ for $1 \mathrm{~min}$. The samples were washed successively with $200 \mu \mathrm{L} 80 \%$ acetone, $180 \mu \mathrm{L}$ acetone and $180 \mu \mathrm{L}$ n-pentane for 1 min at $5000 \mathrm{x} g$ each. The filter was then dried for 2 min under a fume hood to remove all remaining solvents. Afterwards $40 \mu \mathrm{L}$ digestion buffer (1:10 diluted Rapid Digest buffer (Promega, Fitchburg, WI) in water) containing Rapid Digestion Trypsin (Promega) using a protein/enzyme (w/w) ratio of 10:1 was added. The 
filter was incubated for $15 \mathrm{~min}$ in a thermomixer (Thermo Fisher Scientific, Bremen, Germany) at $70^{\circ} \mathrm{C}$ covered by a beaker filled with $70{ }^{\circ} \mathrm{C}$ hot water. After digestion samples were spun down for 1 min at 5000 $\mathrm{x}$ g. The eluted peptide solution was acidified with $10 \mu \mathrm{L} 10 \%$ TFA to a $\mathrm{pH}$ of $\sim 2$. Concentration was measured with the NanoPhotometer® NP80 (Implen, Westlake Village, CA). All samples were diluted with $0.1 \%$ TFA to obtain a final peptide concentration of $0.25 \mu \mathrm{g} / \mu \mathrm{L}$ and stored at $-80^{\circ} \mathrm{C}$.

Liquid chromatography and mass spectrometry. Peptides were analyzed on an EASY-nanoLC 1200 (Thermo Fisher Scientific, Bremen, Germany) coupled online to a Q Exactive ${ }^{\mathrm{TM}}$ Plus mass spectrometer (Thermo Fisher Scientific, Bremen, Germany). $1.25 \mu \mathrm{g}$ peptides were loaded on a $\mu$ PAC ${ }^{\text {TM }}$ trap column (5 $\mu \mathrm{m}$ pillar diameter, $18 \mu \mathrm{m}$ pillar length, 100*2 mm bed channel size, 100-200 A C18, PharmaFluidics, Ghent, Belgium) and then separated on a $\mu \mathrm{PAC}^{\text {TM }}$ analytical column $(5 \mu \mathrm{m}$ pillar diameter, $18 \mu \mathrm{m}$ pillar length, $315 \mu \mathrm{m} * 200 \mathrm{~cm}$ bed channel size, 100-200 ̊̊ C18, PharmaFluidics, Ghent, Belgium) using a stepped 160 min gradient of $80 \%$ acetonitrile (solvent B, Optima LC/MS, Fisher Chemical) in $0.1 \%$ formic acid (solvent A, Optima LC/MS, Fisher Chemical) at $300 \mathrm{~nL} / \mathrm{min}$ flow rate: 3-10\% B in $22 \mathrm{~min}, 10-33 \%$ B in 95 $\min , 33-49 \%$ B in $23 \mathrm{~min}, 49-80 \%$ B in $10 \mathrm{~min}$ and $80 \% \mathrm{~B}$ for $10 \mathrm{~min}$. The $200 \mathrm{~cm} \mu$ PAC $^{\text {TM }}$ column was kept at $50^{\circ} \mathrm{C}$ using a butterfly heater (Phoenix S\&T, Chester, PA. In order to reduce peptide carry-over, 1 $\mu \mathrm{g}$ Candida albicans strain SC5314 digest was loaded on the trap column and subsequently washed using $15 \mu \mathrm{L}$ wash solution (60\% acetonitrile, $30 \%$ isopropanol and $9.9 \%$ water and $0.1 \% \mathrm{FA}$ ) after each sample. During the equilibration step of the next sample, the wash solution that remained in the trap column was then used to wash the analytical column. The Q Exactive ${ }^{\mathrm{TM}}$ Plus was operated in a data-independent (DIA) manner in the $\mathrm{m} / \mathrm{z}$ range of $350-1,150$. Full scan spectra were recorded with a resolution of 70,000 using an automatic gain control (AGC) target value of $3 \times 10^{6}$ with a maximum injection time of $100 \mathrm{~ms}$. The Full scans were followed by 69 DIA scans of dynamic window widths using an overlap of 0.5 Th (See window table in supplement for more information). DIA spectra were recorded at a resolution of 17,500 using an AGC target value of $3 \times 10^{6}$ with a maximum injection time of $55 \mathrm{~ms}$ and a first fixed mass of $200 \mathrm{Th}$. Normalized collision energy (NCE) was set to $25 \%$ and default charge state was set to 3. Peptides were ionized using electrospray with a stainless-steel emitter, I.D. $30 \mu \mathrm{m}$, (Proxeon, Odense, Denmark) at a spray voltage of $2.1 \mathrm{kV}$ and a heated capillary temperature of $275^{\circ} \mathrm{C}$.

Species identification by LC-MS ${ }^{1}$ data. Identification of microbial species was carried out as outlined by (Lasch et al., 2020) with slight modifications. Briefly, peptide $\left(\mathrm{MS}^{1}\right)$ feature lists were extracted from raw DIA data using the Minora algorithm of the Proteome Discoverer ${ }^{\mathrm{TM}}$ software (v2.2.0388, Thermo-Fisher Scientific). The feature lists were pre-processed and subsequently exported into a format compatible with MicrobeMS, a Matlab toolbox (Matlab: The Mathworks, Natick, MA) developed in house for microorganism identification by mass spectrometry (http://wiki.microbe-ms.com). Using MicrobeMS, MS ${ }^{1}$ feature lists were compared against a library of strain-specific in silico mass profiles obtained from 
UniProtKB/Swiss-Prot and UniProtKB/TrEMBL protein sequence data. Ranking lists of correlation, or inter-spectral distance values (i.e. of scores) were obtained that provided information on the taxonomic identity of the organism under study (please see Lasch et al, for details). The in-silico database (v2.0) consisted of 8580 strain-specific in silico profiles, each containing tens of thousands of peptide mass entries. As distance values, variance-scaled Pearson's product momentum correlation coefficients (Pareto scaling 0.25) were selected, whereby data between 2000 and 4500 Da served as inputs. The results of correlation analysis were scored between 0 (no correlation) and 1000 (identity) and arranged in ranking lists to determine the taxonomic identity of the samples investigated.

Peptide identification. The reference proteomes with the highest completeness according to their BUSCO values (Waterhouse et al, 2018) and with a full genome representation were downloaded from UniProtKB for each of the 7 microbial species. AMR-related proteins in this species-specific databases, were then removed using BLASTp algorithm implemented in DIAMOND (v0.9.29, https://github.com/bbuchfink/diamond) (Buchfink et al, 2014) with a sequence identity threshold of $95 \%$ with respect to any entry of the Comprehensive Antibiotic Resistance Database (CARD v3.0.5, September 2019)(Alcock et al., 2020). This modified proteome FASTA is then used as a species-specific background database for detecting AMR-related proteins after merging the databases with all CARD entries. The headers for each protein entry of the CARD, were modified to match our workflow resulting in the following motif: ">CD|Protein name|ARO:XXXXXXX GN=ARO:XXXXXXX". All LC-MS/MS raw data files were analyzed using the species-specific background databases merged with either the whole unfiltered CARD called "Full CARD" (196.072 entries) or a "Whitelisted CARD" (22 entries), which contains only the protein isoforms, targeted previously by PCR (CMY-2, CTX-M-9/15, KPC-2/3, NDM-1, OXA-1/48, SHV1/12, TEM-1/52, VIM-1, AAC(6)-lb-cr, VanA-R/S and VanB-R/S). Those protein isoforms were extracted from the Full CARD using Geneious Prime ${ }^{\circledR}$ (v2020.0.5) and consensus sequences were generated by multiple sequence alignment (MSA) using MAFFT (v6.240, https://mafft.cbrc.jp/)(Katoh \& Standley, 2013; Rozewicki et al, 2019).

Peptides in the LC-MS/MS data were identified using DIA-NN (v1.76) (Demichev et al., 2019). Samples were analyzed separately for each species in an unbatched manner in conjunction with the unrelated run option. Spectral libraries were predicted using the deep-learning algorithm implemented in DIA-NN with strict trypsin specificity (KR not $\mathrm{P}$ ) allowing up to one missed cleavage site in the $\mathrm{m} / \mathrm{z}$ range of $350-1,150$ for all peptides consisting of 7-30 amino acids of the sample-specific background database and either the "Full" or "Whitelisted" version of CARD. Cysteine residues were assumed to be carbamidomethylated and protein $\mathrm{N}$-terminal methionines were removed. The mass spectra were analyzed using fixed mass tolerances of $10 \mathrm{ppm}$ for $\mathrm{MS}^{1}$ and $20 \mathrm{ppm}$ for $\mathrm{MS}^{2}$ spectra. Retention time profiling was disabled. The false discovery 
rate was set to $1 \%$ for precursor identifications and proteins were grouped according to their respective genes.

Antimicrobial resistance detection and reporting. AMR protein families and isoforms as well as the associated drug classes were detected from the DIA-NN output files using the R (v3.6.0) script rawDIAtect developed in house (v0.3, https://github.com/CptChiler/rawDIAtect), which consists of two modules: rawSplit and the "Clinical Resistance Identification Tool" (CRIT). The first module rawSplit, was used to split the peptide result file from DIA-NN into single files for each sample. These files were then loaded into the module CRIT, which extracted all peptides identified from CARD and annotated these hits with antimicrobial resistance related information such as AMR protein family, drug class and AMR protein subfamily deposited in the ARO index file of CARD Ontology (v3.0.5, September 2019) (Alcock et al., 2020). Peptide sequences were then filtered for uniqueness with respect to AMR protein families and for removal of multiple charge states keeping only the charge state with the highest precursor quantity. AMR protein families were inferred from the remaining peptides and were subsequently filtered requiring a minimum of 3 sequences per protein. After this, the abundance of each AMR protein family was calculated from the top 3 precursor intensities (Grossmann et al, 2010; Silva et al, 2006). The most likely AMR protein isoforms were inferred from ranking all identified isoforms per AMR protein family according to their peptide counts. Protein isoforms of one AMR protein family were matched to their subfamily according to the Beta-lactamase database (BLDB) (Naas et al, 2017) and given a new tag "Subfamily" to clear up multiple isoform hits. All findings were collected into a table and visualized with the R package ggplot 2 . All plots were summarized in a single pdf file and the processed peptide tables were separately saved for further investigation (uploaded on Pride). 


\section{Acknowledgments}

We thank Sibylle Müller-Bertling, Kirstin Ganske and Kathleen Klaper for excellent technical and microbiological assistance. We also thank Stephan Fuchs for his support writing this paper.

\section{Author contributions}

J.D., A.S., C.B. and P.L. conceived the study and developed the method.

C.B., J.D. and P.L. designed the experiments.

C.B., C.J. and J.D, performed the proteomic experiments.

Analysis of proteomic data was done by C.B., J.D. and P.L.

C.B. developed the $\mathrm{R}$ package rawDIAtect.

J.D., YP and GW performed the characterization of the isolates (AST, PCR, Sanger sequencing).

C.B., P.L, J.D., W.G. and Y.P. analyzed the study data and wrote the manuscript.

\section{Competing interests}

J.D., A.S. and P.L. are the inventors of SPEED and have submitted a patent application related to SPEED. The other authors declare that they have no competing interests.

\section{Data Availability}

\section{Access to proteomics data}

The mass spectrometry proteomics data have been deposited to the ProteomeXchange Consortium (http://proteomecentral.proteomexchange.org) via the PRIDE partner repository with the dataset identifiers

PXD022425. For access to data contact us

\section{Access to genomic data}

All used genomic data have been deposited at the EMBL-EBI database (https://www.ebi.ac.uk/) and can be found under the access ID ERP123377.

\section{Access to code and programs}

The R-code of rawDIAtect is available on https://github.com/CptChiler/rawDIAtect. MicrobeMS can be downloaded from https://www.microbe-ms.com/ . 
bioRxiv preprint doi: https://doi.org/10.1101/2020.11.17.386540; this version posted December 10, 2020. The copyright holder for this preprint (which was not certified by peer review) is the author/funder. All rights reserved. No reuse allowed without permission.

\section{References}

\section{Footnotes}




\section{Tables}

\begin{tabular}{|c|c|c|c|c|c|c|}
\hline ID & Species & $\mathrm{ST}^{2}$ & AMR determinants (PCR and Sanger sequencing) & Antibiotic Resistances (BMD, Etest, VITEK 2 AST N248; EUCAST v10.0) & Sample type & Reference \\
\hline $604-16$ & Citrobacter freundii & ST98 & KPC-2,TEM-1,OXA-1,AAC( $\left.6^{\circ}\right)-\mathrm{Ib}-\mathrm{cr}$ & AMP,PIP,PTZ,ATM,CTX,CAZ,FOX,CEF,MEM,ETP,IPM,GEN,TOB,MOX,NAL,CIP,CMP,SXT & Clinical & (Schweizer et al, 2019) \\
\hline $151-09$ & $\begin{array}{l}\begin{array}{l}\text { Enterobacter } \\
\text { hormaechei }\end{array} \\
\end{array}$ & n.t. & VIM-1 & AMP,PIP,PTZ,CTX,CAZ,FOX,CEF,MEM,ETP,IPM,TOB,AMK,MOX,NAL,CIP,CMP,SXT,CST,FOS,TGC & Clinical & \\
\hline UW12819 & $\begin{array}{l}\text { Enterococcus } \\
\text { faecium }\end{array}$ & ST117 & - & STR(HL), ERY & Clinical & \\
\hline UW14883 & $\begin{array}{l}\text { Enterococcus } \\
\text { faecium }\end{array}$ & ST80 & - & STR(HL), ERY & Clinical & \\
\hline UW16369 & $\begin{array}{l}\text { Enterococcus } \\
\text { faecium }\end{array}$ & ST262 & - & ERY, TET & Clinical & \\
\hline UW17739 & $\begin{array}{l}\text { Enterococcus } \\
\text { faecium }\end{array}$ & ST612 & VanA & AMP, VAN, TEI, GEN(HL), ERY, CIP, TET & Clinical & \\
\hline UW19605 & $\begin{array}{l}\text { Enterococcus } \\
\text { faecium }\end{array}$ & ST80 & VanA & AMP, VAN, TEI, GEN(HL), ERY, CIP, RAM & Clinical & \\
\hline UW14142 & $\begin{array}{l}\text { Enterococcus } \\
\text { faecium }\end{array}$ & ST117 & VanA & AMP, VAN, TEI, ERY, CIP, SXT & Clinical & \\
\hline UW16517 & $\begin{array}{l}\text { Enterococcus } \\
\text { faecium }\end{array}$ & ST80 & VanB & AMP, VAN, STR(HL), ERY, CIP & Clinical & \\
\hline UW18273 & $\begin{array}{l}\text { Enterococcus } \\
\text { faecium }\end{array}$ & ST117 & VanB & AMP, VAN, ERY, CIP & Clinical & \\
\hline UW12913 & $\begin{array}{l}\text { Enterococcus } \\
\text { faecium }\end{array}$ & ST17 & VanB & AMP, VAN, GEN(HL), STR(HL), ERY, CMP, CIP & Clinical & \\
\hline $531-12 \_\mathrm{K} 4$ & Escherichia coli & ST10 & CMY-2 & AMP,PIP,CTX,CAZ,FOX & 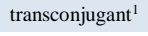 & (Pietsch et al, 2017) \\
\hline 330-16_K1 & Escherichia coli & ST10 & MCR-1 & CST (I) & 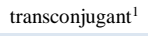 & \\
\hline 151-09_K1 & Escherichia coli & ST10 & VIM-1 & AMP,PIP,PTZ,CTX,CAZ,FOX,CEF (I),MEM,ETP,IPM (I),TOB,AMK,SXT & 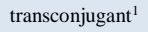 & \\
\hline 16-10_K1 & Escherichia coli & ST10 & OXA-48 & AMP,PIP,PTZ & 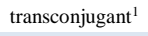 & (Pfeifer et al, 2012) \\
\hline 443-16_K1 & Escherichia coli & ST10 & CTX-M-9 & AMP,PIP,ATM,CTX,CAZ,CEF (I) & 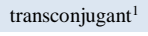 & \\
\hline 58-07_K1 & Escherichia coli & ST10 & SHV-12 & AMP,PIP,ATM CAZ & 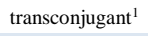 & (Eller et al, 2013) \\
\hline 17-11_K1 & Escherichia coli & ST10 & KPC-2 & AMP, PIP, PTZ, ATM, CTX, CAZ (I), FOX, MEM, ETP, IPM (I), GEN, TOB, AMK,CMP & 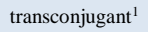 & \\
\hline 371-12_K1 & Escherichia coli & ST10 & NDM-7 & AMP PIP,PTZ,CTX,CAZ,FOX,CEF,MEM,ETP,IPM & 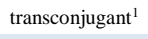 & \\
\hline E.c._J53Azir & Escherichia coli & ST10 & - & - & recipient & (Clowes \& Rowley, 1954) \\
\hline $281-15$ & $\begin{array}{l}\begin{array}{l}\text { Klebsiella } \\
\text { pneumoniae }\end{array} \\
\end{array}$ & ST258 & KPC-2,SHV-11,TEM-1, OXA-9 $9^{3}, \mathrm{AAC}(6)-\mathrm{Ib}$ & AMP,PIP, PTZ,ATM,CTX,CAZ,CEF (I),MEM,ETP,IPM (I),GEN,TOB,AMK,MOX,NAL,CIP,CMP,SXT,TGC & Clinical & (Becker et al., 2018) \\
\hline $292-15$ & $\begin{array}{l}\text { Klebsiella } \\
\text { pneumoniae }\end{array}$ & ST258 & KPC-3,SHV-11,TEM-1, OXA-9 $9^{3}, \mathrm{AAC}(6)-\mathrm{Ib}$ & AMP,PIP,PTZ,ATM,CTX,CAZ,CEF,MEM,ETP,IPM,GEN,TOB,AMK,MOX,NAL,CIP,CMP,SXT,FOS,TGC & Clinical & (Becker et al., 2018) \\
\hline $302-15$ & $\begin{array}{l}\text { Klebsiella } \\
\text { pneumoniae }\end{array}$ & ST629 & OXA-48,SHV-11,CTX-M-15,TEM-1,AAC(6')-Ib-cr & AMP,PIP,PTZ,ATM,CTX,CAZ,MEM (I),ETP,IPM (I),GEN,TOB,MOX,NAL,CIP,CMP,SXT,FOS,TGC & Clinical & (Becker et al., 2018) \\
\hline $276-15$ & $\begin{array}{l}\text { Klebsiella } \\
\text { pneumoniae }\end{array}$ & ST101 & KPC-2,SHV-1,CTX-M-15,TEM-1,OXA-9 $9^{3}, \mathrm{AAC}(6)-\mathrm{Ib}$ & AMP,PIP,PTZ,ATM,CTX,CAZ,CEF,MEM,ETP,IPM,GEN,TOB,AMK,MOX,NAL,CIP,CMP,CST,FOS,TGC & Clinical & (Becker et al., 2018) \\
\hline 229-09 & $\begin{array}{l}\text { Klebsiella } \\
\text { pneumoniae }\end{array}$ & ST147 & $\begin{array}{l}\text { OXA-48,SHV-11,CTX-M-15,TEM-1,OXA-93,AAC(6)- } \\
\text { Ib }\end{array}$ & AMP,PIP,PTZ,ATM,CTX,CAZ,CEF, MEM,ETP,IPM,GEN,TOB,AMK,MOX,NAL,CIP,FOS & Clinical & (Becker et al., 2018) \\
\hline $272-15$ & $\begin{array}{l}\text { Klebsiella } \\
\text { pneumoniae }\end{array}$ & ST101 & KPC-2,SHV-11,TEM-1,OXA-933 & AMP,PIP,PTZ,ATM,CTX,CAZ,MEM,ETP,IPM,MOX,NAL,CIP,SXT,FOS & Clinical & (Becker et al., 2018) \\
\hline $86-09$ & $\begin{array}{l}\text { Klebsiella } \\
\text { pneumoniae }\end{array}$ & ST258 & KPC-2,SHV-11,TEM-1, OXA-9 $9^{3}$, AAC $\left(6^{\prime}\right)-\mathrm{Ib}$ & AMP,PIP,PTZ,ATM,CTX,CAZ,CEF,MEM,ETP,IPM (I),GEN,TOB,AMK,MOX,NAL,CIP,CMP,CST,TGC & Clinical & (Becker et al., 2018) \\
\hline 50-07 & Salmonella Infantis & n.t. & TEM-52 & AMP,PIP,ATM,CTX,CAZ,CEF (I) & Clinical & (Eller et al., 2013) \\
\hline 58-07 & $\begin{array}{l}\text { Salmonella } \\
\text { Typhimurium }\end{array}$ & n.t. & SHV-12 & AMP,PIP,ATM,CTX,CAZ,SXT & Clinical & (Eller et al., 2013) \\
\hline
\end{tabular}

Table 1: List of bacterial isolates (clinical isolates and transconjugants) with metadata

${ }^{1}$ transconjugant as a result of broth mating using recipient strain E. coli J53 Azi ${ }^{\mathrm{r}}$; ${ }^{2}$ sequence type (ST) according to published multilocus sequence typing (MLST) schemes for E. coli (https://enterobase.warwick.ac.uk/species/ecoli/allele_st_search); K. pneumoniae (https://bigsdb.pasteur.fr/klebsiella/klebsiella.html), C. freundii (https://pubmlst.org/cfreundii ), and E. faecium (https://pubmlst.org/efaecium/); ${ }^{3}$ OXA-9 is non-functional due to premature STOP codon (L112STOP)

Phenotype legend (Broth microdilution according EUCAST v10.0 and VITEK 2 card AST N248): AMP, ampicillin; PIP, piperacillin; PTZ, piperacillin/tazobactam; CTX, cefotaxime, CAZ, ceftazidime;

CEF, cefepime; FOX, cefoxitin; MEM, meropenem; IPM, imipenem; ETP, ertapenem; GEN, gentamicin; TOB, tobramycin; AMK, amikacin; NAL, nalidixic acid; CIP, ciprofloxacin; MOX, moxifloxacin,

trimethoprim-sulfamethoxazole; CST, colistin; FOS, fosfomycin; TEI, teicoplanin; TGC, tigecycline; VAN, vancomycin; (I) susceptible, increased exposure/dosage; n.t. not tested. 


\begin{tabular}{|c|c|c|c|}
\hline Sample ID & Species & Sequence type & LC-MS ${ }^{1}$ biotyping Top hit \\
\hline $604-16$ & Citrobacter freundii & ST98 & Citrobacter freundii complex sp. CFNIH4 (Strain: CFNIH4) \\
\hline $151-09$ & Enterobacter hormaechei & n.t. & Enterobacter hormaechei (Strain: ECC904) \\
\hline E.c.J53 AZI ${ }^{\mathrm{R}}$ & Escherichia coli & ST10 & Escherichia coli O157:H7 (Strain: FWSEC0004) \\
\hline 58-07_K1 & Escherichia coli & ST10 & Escherichia coli O157:H7 (Strain: FWSEC0004) \\
\hline $531-1 \overline{2} \_\mathrm{K} 4$ & Escherichia coli & ST10 & Escherichia coli O157:H7 (Strain: FWSEC0004) \\
\hline $443-16 \_K 1$ & Escherichia coli & ST10 & Shigella boydii serotype 18 (strain CDC 3083-94 / BS512) (Strain: CDC 3083-94 / BS512) \\
\hline 371-12_K1 & Escherichia coli & ST10 & Escherichia coli O157:H7 (Strain: FWSEC0004) \\
\hline $330-16 \_\mathrm{K} 1$ & Escherichia coli & ST10 & Escherichia coli (strain K12) (Strain: K12 / W3110 / ATCC 27325 / DSM 5911) \\
\hline $17-11 \_\overline{\mathrm{K}} 1$ & Escherichia coli & ST10 & Escherichia coli O157:H7 (Strain: FWSEC0004) \\
\hline 16-10_K1 & Escherichia coli & ST10 & Escherichia coli O157:H7 (Strain: FWSEC0004) \\
\hline 151-09_K1 & Escherichia coli & ST10 & Shigella flexneri serotype 5 a (strain M90T) (Strain: M90T / Serotype 5a) \\
\hline UW19605 & Enterococcus faecium & ST80 & Enterococcus faecium EnGen0020 (Strain: E1574) \\
\hline UW18273 & Enterococcus faecium & ST117 & Enterococcus faecium E417 (Strain: E417) \\
\hline UW17739 & Enterococcus faecium & ST612 & Enterococcus faecium OC2A-1 (Strain: OC2A-1) \\
\hline UW16517 & Enterococcus faecium & ST80 & Enterococcus faecium EnGen0020 (Strain: E1574) \\
\hline UW16369 & Enterococcus faecium & ST262 & Enterococcus faecium EnGen0020 (Strain: E1574) \\
\hline UW14883 & Enterococcus faecium & ST80 & Enterococcus faecium E417 (Strain: E417) \\
\hline UW14142 & Enterococcus faecium & ST117 & Enterococcus faecium OC2A-1 (Strain: OC2A-1) \\
\hline UW12913 & Enterococcus faecium & ST17 & Enterococcus faecium OC2A-1 (Strain: OC2A-1) \\
\hline UW12819 & Enterococcus faecium & ST117 & Enterococcus faecium E417 (Strain: E417) \\
\hline $86-09$ & Klebsiella pneumoniae & ST258 & Klebsiella pneumoniae 30684/NJST258_2 (Strain: 30684/NJST258_2) \\
\hline $302-15$ & Klebsiella pneumoniae & ST629 & Klebsiella pneumoniae 30684/NJST258_2 (Strain: 30684/NJST258_2) \\
\hline $292-15$ & Klebsiella pneumoniae & ST258 & Klebsiella pneumoniae 30684/NJST258_2 (Strain: 30684/NJST258_2) \\
\hline $281-15$ & Klebsiella pneumoniae & ST258 & $\begin{array}{l}\text { Klebsiella pneumoniae subsp. pneumoniae (strain ATCC } 700721 \text { / MGH 78578) (Strain: ATCC } 700721 \text { / } \\
\text { MGH 78578) }\end{array}$ \\
\hline $276-15$ & Klebsiella pneumoniae & ST101 & $\begin{array}{l}\text { Klebsiella pneumoniae subsp. pneumoniae (strain ATCC } 700721 \text { / MGH 78578) (Strain: ATCC } 700721 \text { / } \\
\text { MGH 78578) }\end{array}$ \\
\hline $272-15$ & Klebsiella pneumoniae & ST101 & $\begin{array}{l}\text { Klebsiella pneumoniae subsp. pneumoniae (strain ATCC } 700721 \text { / MGH 78578) (Strain: ATCC } 700721 \text { / } \\
\text { MGH 78578) }\end{array}$ \\
\hline 229-09 & Klebsiella pneumoniae & ST147 & Klebsiella pneumoniae subsp. pneumoniae (Strain: RJA166) \\
\hline $50-07$ & $\begin{array}{l}\text { Salmonella enterica spp. enterica ser. } \\
\text { Infantis }\end{array}$ & n.t. & Salmonella enterica subsp. enterica serovar Adelaide (Strain: FSIS11808911) \\
\hline $58-07$ & $\begin{array}{l}\text { Salmonella enterica spp. enterica ser. } \\
\text { Typhimurium }\end{array}$ & n.t. & Salmonella typhimurium (strain LT2 / SGSC1412 / ATCC 700720) (Strain: LT2 / SGSC1412 / ATCC 700720) \\
\hline
\end{tabular}

The top hit of the LC-MS ${ }^{1}$ biotyping analysis for each sample is listed. Green marked hits are correct bacterial species identifications. ; n.t. not tested
Thes 


\section{Figures}

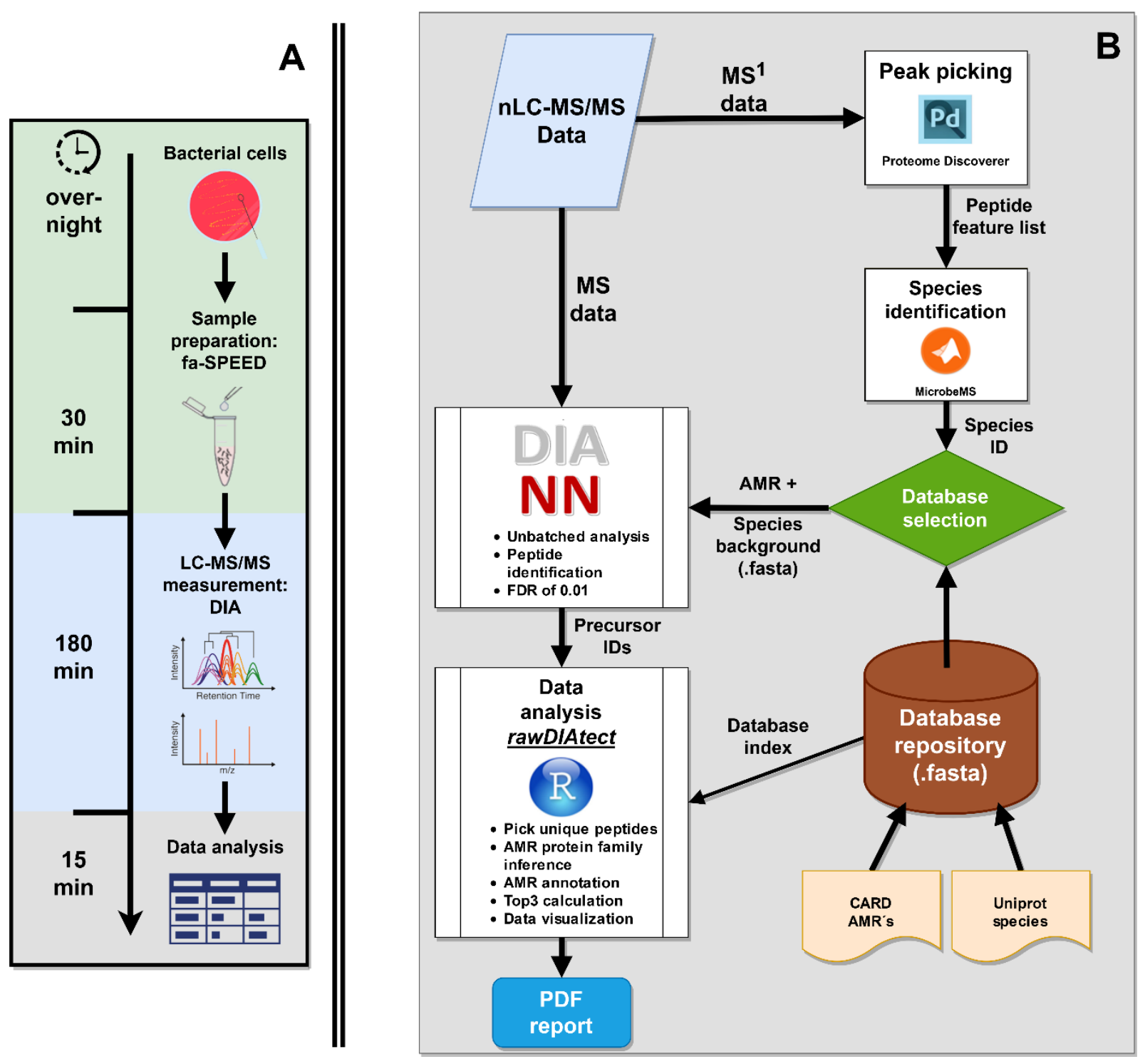

Figure 1: Proteomic workflow of species identification and antimicrobial resistance detection

A: Timeline of the whole workflow with cultivation overnight and sample preparation by the fa-SPEED protocol (green). Peptides were measured with a nanoLC setup coupled to a Orbitrap MS system in a data independent manner (blue) followed by the data analysis (gray).

B: Overview of the data analysis workflow. The $\mathrm{MS}^{1}$ data is used for peak picking in the Proteome Discoverer ${ }^{\mathrm{TM}}$ using the Minora algorithm. The resulting feature list is then used for species identification with MicrobeMS. The species ID is used to select a species background database from UniProt. This species background database is combined with the CARD AMR database. The combined fasta was put into DIA-NN to analyze the $\mathrm{MS}^{2}$ data resulting in a list of found peptides. This peptide hit list is then analyzed by the R script rawDIAtect to detect AMR's which give a pdf report of all found resistances. 


\section{Full CARD database search}






\section{Specificity (Proteomics/Whitelisted CARD) Sensitivity (Proteomics/Whitelisted CARD) Sensitivity (Proteomics/Full CARD)}

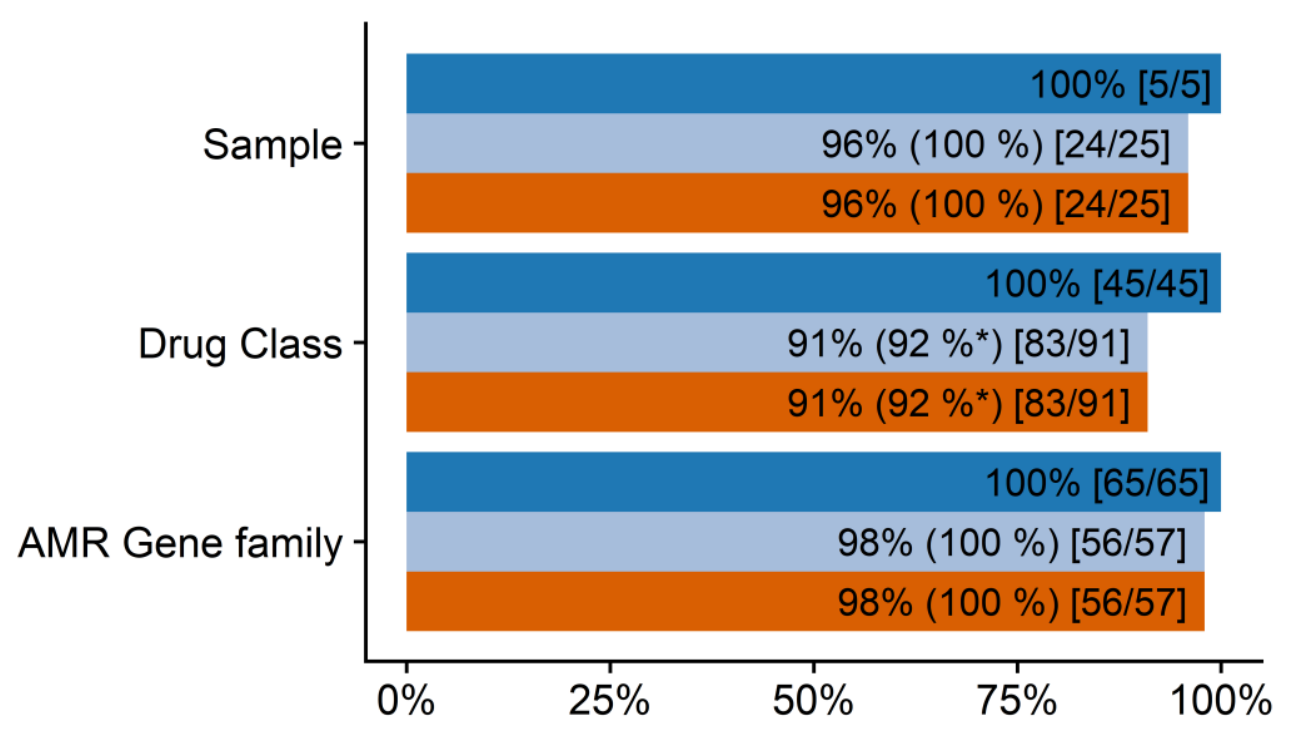

Figure 3: Sensitivity / specificity of AMR detection by proteomics at the sample, drug class and AMR gene level Sensitivity was calculated by the formula TP/(TP + FN) using the results of the Full and Whitelisted CARD in comparison to PCR/Sanger sequencing. Specificity was calculated according to TN / (TN + FP) using the results of the Whitelisted CARD search in comparison to PCR/sanger sequencing and NGS tools Abricate (https://github.com/tseemann/abricate, v1.0.1) and Kleborate

(https://github.com/katholt/Kleborate, v1.0.0). The sensitivity value in the bracket is calculated after inference of vancomycin resistance by the presence of the van ligase regulatory system (see results and discussion for details). TP/FP - true, or false positive assignment, respectively. TN/FN - true, or false negative assignment.

Definition of TP and TN at the sample level: A sample was considered as TP only in cases where all AMR protein families (as determined by PCR/Sanger sequencing) were correctly assigned (FN otherwise). Vice versa, a sample was defined as TN at the sample level if no AMR family was determined in the control samples (FP otherwise). One false negative (FN) assignments was observed (missed VanB detection, see text for details).

TP and TN at the drug class level: A found drug class, was counted as TP, if matched to a phenotype of the sample. 91 drug classes hits over all measurements were observed. One FN, the missing vancomycin hit was observed and 7 predictions can only be potently matched due to combination of multiple resistances. A TN was only counted if no drug class was found. In total 9 drug classes could be found per negative measurement. * Prediction of Drug class considering all possible protein isoform hits including vancomycin.

TP / TN at the gene family level: A TP was counted for every correct AMR gene family hit given by PCR and missing hits as FN. One missing VanB family hit was observed. In total 57 AMR gene family hits could be found over all measurements. TN was only counted if no hit could be found for any possible AMR determinant (13) per negative measurement. 


\section{Whitelisted CARD search}

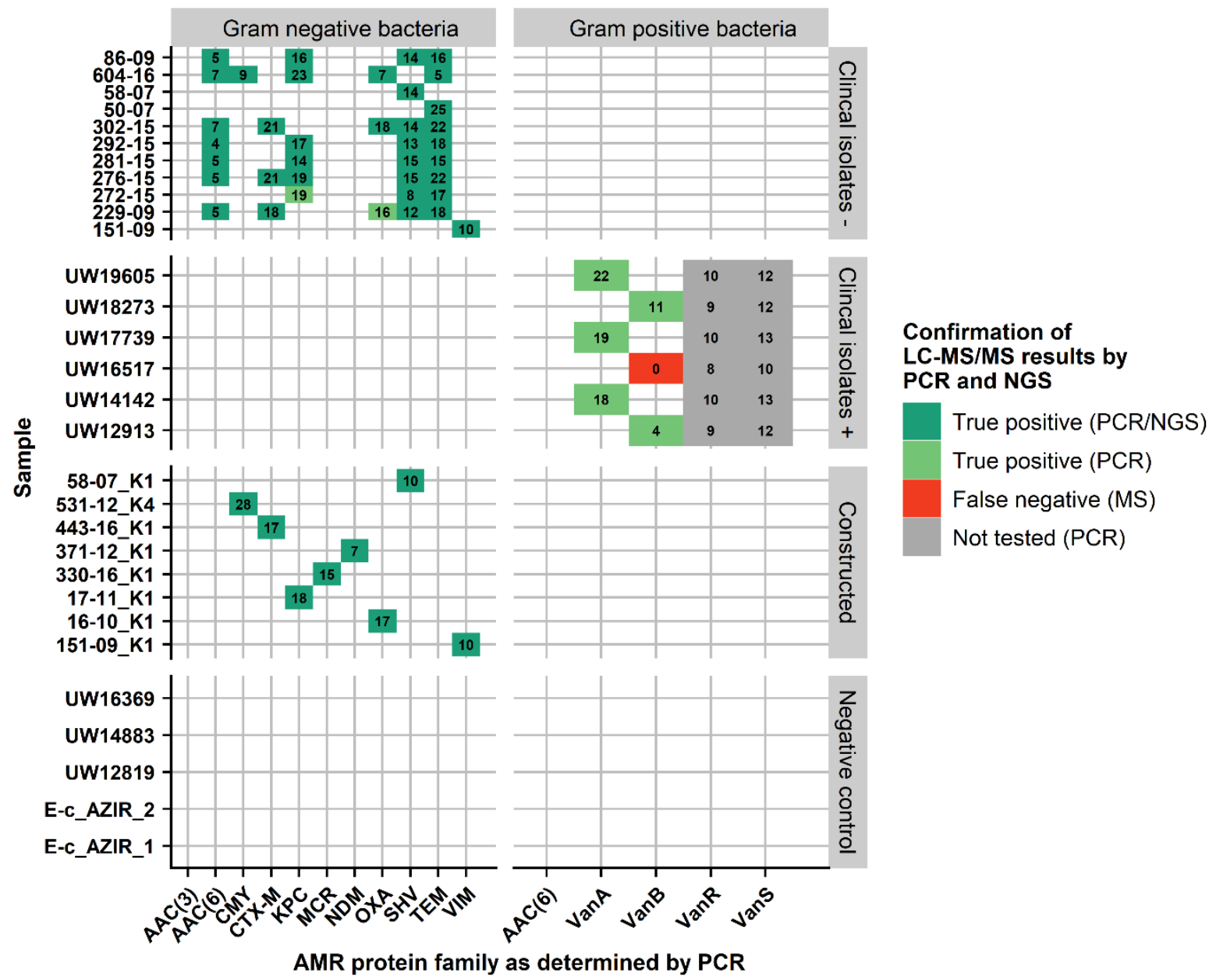

Figure 4: Comparison of AMR protein family detected by proteomics using Whitelisted CARD search approved by PCR/sanger sequencing and NGS as the reference method

Proteomic hits verified by PCR and NGS are plotted in dark green. Additional hits of proteomics, which were verified only by PCR are plotted in light green and missing hits are plotted in red. The numbers stand for the count of unique peptides detected by MS. One missing hit was the Van ligase B, however proteomics was able to detect the corresponding components of the regulatory system VanR and VanS, which were not analyzed by PCR (Gray). This two-component system was detected in all VanB and VanA positive E. faecium samples and confirms the presence of a van ligase by the molecular function of the van gene cluster. No additional hits of proteomics, which could not be confirmed by either PCR or NGS (Abricate, https://github.com/tseemann/abricate, v1.0.1 and Kleborate, https://github.com/katholt/Kleborate, v1.0.0) were detected. 


\section{References}

Alcock BP, Raphenya AR, Lau TTY, Tsang KK, Bouchard M, Edalatmand A, Huynh W, Nguyen A-LV, Cheng AA, Liu S et al (2020) CARD 2020: antibiotic resistome surveillance with the comprehensive antibiotic resistance database. Nucleic Acids Res 48: 517--525

Bache N, Geyer PE, Bekker-Jensen DB, Hoerning O, Falkenby L, Treit PV, Doll S, Paron I, Müller JB, Meier F et al (2018) A Novel LC System Embeds Analytes in Pre-formed Gradients for Rapid, Ultrarobust Proteomics. Molecular \& Cellular Proteomics 17: 2284--2296

Becker L, Kaase M, Pfeifer Y, Fuchs S, Reuss A, von Laer A, Sin MA, Korte-Berwanger M, Gatermann S, Werner G (2018) Genome-based analysis of Carbapenemase-producing Klebsiella pneumoniae isolates from German hospital patients, 2008-2014. Antimicrob Resist Infect Control 7

Bhattacharyya RP, Bandyopadhyay N, Ma P, Son SS, Liu J, He LL, Wu L, Khafizov R, Boykin R, Cerqueira GC et al (2019) Simultaneous detection of genotype and phenotype enables rapid and accurate antibiotic susceptibility determination. Nat Med 25: 1858--1864

Buchfink B, Xie C, Huson DH (2014) Fast and sensitive protein alignment using DIAMOND. Nat Methods 12: 59--60

Cecchini T, Yoon E-J, Charretier Y, Bardet C, Beaulieu C, Lacoux X, Docquier J-D, Lemoine J, Courvalin P, Grillot-Courvalin C et al (2018) Deciphering Multifactorial Resistance Phenotypes in $<\mathrm{i}>$ Acinetobacter baumannii $</ \mathrm{i}>$ by Genomics and Targeted Label-free Proteomics. PubMed comprises more than 30 million citations for biomedical literature from MEDLINE, life science journals, and online books 17: 442--456

Charretier Y, Dauwalder O, Franceschi C, Degout-Charmette E, Zambardi G, Cecchini T, Bardet C, Lacoux X, Dufour P, Veron L et al (2015) Rapid Bacterial Identification, Resistance, Virulence and Type Profiling using Selected Reaction Monitoring Mass Spectrometry. Sci Rep 5: 13944

Chen C-Y, Clark CG, Langner S, Boyd DA, Bharat A, McCorrister SJ, McArthur AG, Graham MR, Westmacott GR, Van Domselaar G (2019) Detection of Antimicrobial Resistance Using Proteomics and the Comprehensive Antibiotic Resistance Database: A Case Study. Proteomics Clin Appl e1800182

Clowes RC, Rowley D (1954) Some Observations on Linkage Effects in Genetic Recombination in Escherichia coli K-12. Microbiology 11: 250--260

Correa-Martínez CL, Idelevich EA, Sparbier K, Kostrzewa M, Becker K (2019) Rapid Detection of Extended-Spectrum $\beta$-Lactamases (ESBL) and AmpC $\beta$-Lactamases in Enterobacterales: Development of a Screening Panel Using the MALDI-TOF MS-Based Direct-on-Target Microdroplet Growth Assay. Front Microbiol 10

Demichev V, Messner CB, Vernardis SI, Lilley KS, Ralser M (2019) DIA-NN: neural networks and interference correction enable deep proteome coverage in high throughput. Nat Methods 17: 41--44

Doellinger J, Blumenscheit C, Schneider A, Lasch P (2020a) Isolation Window Optimization of DataIndependent Acquisition Using Predicted Libraries for Deep and Accurate Proteome Profiling. Anal Chem 
Doellinger J, Schneider A, Hoeller M, Lasch P (2020b) Sample Preparation by Easy Extraction and Digestion (SPEED) - A Universal, Rapid, and Detergent-free Protocol for Proteomics Based on Acid Extraction. Molecular \& Cellular Proteomics 19: 209--222

Dubourg G, Lamy B, Ruimy R (2018) Rapid phenotypic methods to improve the diagnosis of bacterial bloodstream infections: meeting the challenge to reduce the time to result. Clinical Microbiology and Infection 24: 935--943

Eller C, Simon S, Miller T, Frick J-S, Prager R, Rabsch W, Guerra B, Werner G, Pfeifer Y (2013) Presence of $\beta$-lactamases in extended-spectrum-cephalosporin-resistant Salmonella enterica of 30 different serovars in Germany 2005-11. J Antimicrob Chemother 68: 1978--1981

Foudraine DE, Dekker LJM, Strepis N, Bexkens ML, Klaassen CHW, Luider TM, Goessens WHF (2019) Accurate Detection of the Four Most Prevalent Carbapenemases in E. coli and K. pneumoniae by HighResolution Mass Spectrometry. Front Microbiol 10

Galimand M, Schmitt E, Panvert M, Desmolaize B, Douthwaite S, Mechulam Y, Courvalin P (2011) Intrinsic resistance to aminoglycosides in Enterococcus faecium is conferred by the 16S rRNA m5C1404specific methyltransferase EfmM. RNA 17: 251

Gröbner S, Linke D, Schütz W, Fladerer C, Madlung J, Autenrieth IB, Witte W, Pfeifer Y (2009) Emergence of carbapenem-non-susceptible extended-spectrum beta-lactamase-producing Klebsiella pneumoniae isolates at the university hospital of Tübingen, Germany. J Med Microbiol 58: 7

Grossmann J, Roschitzki B, Panse C, Fortes C, Barkow-Oesterreicher S, Rutishauser D, Schlapbach R (2010) Implementation and evaluation of relative and absolute quantification in shotgun proteomics with label-free methods. J Proteomics 73: 1740--1746

Hassing R-J, Goessens WH, Zeneyedpour L, Sultan S, van Kampen JJ, Verbon A, van Genderen PJ, Hays JP, Luider TM, Dekker LJ (2016) Detection of amino acid substitutions in the GyrA protein of fluoroquinolone-resistant typhoidal Salmonella isolates using high-resolution mass spectrometry. Int $J$ Antimicrob Agents 47: 351--356

Hollenbeck BL, Rice LB (2012) Intrinsic and acquired resistance mechanisms in enterococcus. Virulence 3: 421

Hou T-Y, Chiang-Ni C, Teng S-H (2019) Current status of MALDI-TOF mass spectrometry in clinical microbiology. J Food Drug Anal 27: 404--414

Katoh K, Standley DM (2013) MAFFT multiple sequence alignment software version 7: improvements in performance and usability. Mol Biol Evol 30: 772-780

Khaledi A, Schniederjans M, Pohl S, Rainer R, Bodenhofer U, Xia B, Klawonn F, Bruchmann S, Preusse M, Eckweiler D et al (2016) Transcriptome Profiling of Antimicrobial Resistance in Pseudomonas aeruginosa. Antimicrob Agents Chemother 60: 4722--4733

Khot PD, Fisher MA (2013) Novel Approach for Differentiating Shigella Species and Escherichia coli by Matrix-Assisted Laser Desorption Ionization-Time of Flight Mass Spectrometry. J Clin Microbiol 51: 3711--3716

Lange C, Schubert S, Jung J, Kostrzewa M, Sparbier K (2014) Quantitative matrix-assisted laser desorption ionization-time of flight mass spectrometry for rapid resistance detection. J Clin Microbiol 52: 4155--4162 
Lasch P, Schneider A, Blumenscheit C, Doellinger J (2020) Identification of Microorganisms by Liquid Chromatography-Mass Spectrometry (LC-MS1) and in silico Peptide Mass Libraries. Molecular \& Cellular Proteomics

Liu Y-Y, Wang Y, Walsh TR, Yi L-X, Zhang R, Spencer J, Doi Y, Tian G, Dong B, Huang X et al (2016) Emergence of plasmid-mediated colistin resistance mechanism MCR-1 in animals and human beings in China: a microbiological and molecular biological study. Lancet Infect Dis 16: 161--168

Meier F, Brunner A-D, Koch S, Koch H, Lubeck M, Krause M, Goedecke N, Decker J, Kosinski T, Park MA et al (2018) Online parallel accumulation - serial fragmentation (PASEF) with a novel trapped ion mobility mass spectrometer. Molecular \& Cellular Proteomics

Messner CB, Demichev V, Bloomfield N, White M, Kreidl M, Ivosev G, Wasim F, Zelezniak A, Lilley KS, Tate $\mathrm{S}$ et al (2020) Scanning SWATH acquisition enables high-throughput proteomics with chromatographic gradients as fast as 30 seconds. bioRxiv: 656793

Naas T, Oueslati S, Bonnin RA, Dabos ML, Zavala A, Dortet L, Retailleau P, Iorga BI (2017) Betalactamase database (BLDB) - structure and function. J Enzyme Inhib Med Chem 32: 917--919

Nguyen M, Long SW, McDermott PF, Olsen RJ, Olson R, Stevens RL, Tyson GH, Zhao S, Davis JJ (2019) Using Machine Learning To Predict Antimicrobial MICs and Associated Genomic Features for Nontyphoidal Salmonella. J Clin Microbiol 57

Nix ID, Idelevich EA, Storck LM, Sparbier K, Drews O, Kostrzewa M, Becker K (2020) Detection of Methicillin Resistance in Staphylococcus aureus From Agar Cultures and Directly From Positive Blood Cultures Using MALDI-TOF Mass Spectrometry-Based Direct-on-Target Microdroplet Growth Assay. Front Microbiol 11

O. n. s. o. r. t. i. u. m. UniProt C (2019) UniProt: a worldwide hub of protein knowledge. Nucleic Acids Res 47: 506--515

O’Neill J (2016) TACKLING DRUG-RESISTANT INFECTIONS GLOBALLY: FINAL REPORT AND RECOMMENDATIONS.

Paauw A, Jonker D, Roeselers G, Heng JME, Mars-Groenendijk RH, Trip H, Molhoek EM, Jansen H-J, van der Plas J, de Jong AL et al (2015) Rapid and reliable discrimination between Shigella species and Escherichia coli using MALDI-TOF mass spectrometry. Int J Med Microbiol 305: 446--452

Park CH, Robicsek A, Jacoby GA, Sahm D, Hooper DC (2006) Prevalence in the United States of aac(6')Ib-cr encoding a ciprofloxacin-modifying enzyme. Antimicrob Agents Chemother 50: 3953--3955

Patel R (2015) MALDI-TOF MS for the diagnosis of infectious diseases. Clin Chem 61: 100--111

Pfeifer Y, Schlatterer K, Engelmann E, Schiller RA, Frangenberg HR, Stiewe D, Holfelder M, Witte W, Nordmann P, Poirel L (2012) Emergence of OXA-48-Type Carbapenemase-Producing

Enterobacteriaceae in German Hospitals. Antimicrob Agents Chemother 56: 2125

Pfeifer Y, Wilharm G, Zander E, Wichelhaus TA, Göttig S, Hunfeld K-P, Seifert H, Witte W, Higgins PG (2011) Molecular characterization of blaNDM-1 in an Acinetobacter baumannii strain isolated in Germany in 2007. J Antimicrob Chemother 66: 1998--2001

Pietsch M, Eller C, Wendt C, Holfelder M, Falgenhauer L, Fruth A, Grössl T, Leistner R, Valenza G, Werner G et al (2017) Molecular characterisation of extended-spectrum $\beta$-lactamase (ESBL)-producing 
Escherichia coli isolates from hospital and ambulatory patients in Germany. Veterinary Microbiology 200: 130--137

Rozewicki J, Li S, Amada KM, Standley DM, Katoh K (2019) MAFFT-DASH: integrated protein sequence and structural alignment. Nucleic Acids Res 47: W5--W10

Schweizer C, Bischoff P, Bender J, Kola A, Gastmeier P, Hummel M, Klefisch F-R, Schoenrath F, Frühauf A, Pfeifer Y (2019) Plasmid-Mediated Transmission of KPC-2 Carbapenemase in Enterobacteriaceae in Critically Ill Patients. Front Microbiol 10

Silva JC, Gorenstein MV, Li G-Z, Vissers JPC, Geromanos SJ (2006) Absolute quantification of proteins by LCMSE: a virtue of parallel MS acquisition. PubMed comprises more than 30 million citations for biomedical literature from MEDLINE, life science journals, and online books 5: 144--156

Sparbier K, Schubert S, Kostrzewa M (2016) MBT-ASTRA: A suitable tool for fast antibiotic susceptibility testing? PubMed comprises more than 30 million citations for biomedical literature from MEDLINE, life science journals, and online books 104: 48--54

Strich JR, Wang H, Cissé OH, Youn J-H, Drake SK, Chen Y, Rosenberg AZ, Gucek M, McGann PT, Dekker JP et al (2019) Identification of the OXA-48 Carbapenemase Family by Use of Tryptic Peptides and Liquid Chromatography-Tandem Mass Spectrometry. J Clin Microbiol 57: 5

Su M, Satola SW, Read TD (2019) Genome-Based Prediction of Bacterial Antibiotic Resistance. J Clin Microbiol 57

Turton JF, Woodford N, Glover J, Yarde S, Kaufmann ME, Pitt TL (2006) Identification of Acinetobacter baumannii by detection of the blaOXA-51-like carbapenemase gene intrinsic to this species. J Clin Microbiol 44: 2974--2976

van Belkum A, Burnham C-AD, Rossen JWA, Mallard F, Rochas O, Dunne WM (2020) Innovative and rapid antimicrobial susceptibility testing systems. Nat Rev Microbiol 18: 299--311

van Belkum A, Dunne WM (2013) Next-Generation Antimicrobial Susceptibility Testing. J Clin Microbiol 51: 2018--2024

van Veen SQ, Claas ECJ, Kuijper EJ (2010) High-throughput identification of bacteria and yeast by matrix-assisted laser desorption ionization-time of flight mass spectrometry in conventional medical microbiology laboratories. J Clin Microbiol 48: 900--907

Waterhouse RM, Seppey M, Simão FA, Manni M, Ioannidis P, Klioutchnikov G, Kriventseva EV, Zdobnov EM (2018) BUSCO Applications from Quality Assessments to Gene Prediction and Phylogenomics. Mol Biol Evol 35: 543--548

Welker M, van Belkum A (2019) One System for All: Is Mass Spectrometry a Future Alternative for Conventional Antibiotic Susceptibility Testing? Front Microbiol 10

Werner G, Klare I, Fleige C, Geringer U, Witte W, Just H-M, Ziegler R (2012) Vancomycin-resistant vanB-type Enterococcus faecium isolates expressing varying levels of vancomycin resistance and being highly prevalent among neonatal patients in a single ICU. Robert Koch-Institut, Infektionskrankheiten / Erreger

WHO (2014) ANTIMICROBIAL RESISTANCE: Global Report on Surveillance. WHO Report 
bioRxiv preprint doi: https://doi.org/10.1101/2020.11.17.386540; this version posted December 10, 2020. The copyright holder for this preprint (which was not certified by peer review) is the author/funder. All rights reserved. No reuse allowed without permission.

WHO (2015) GLOBAL ACTION PLAN on antimicrobial resistance. WHO Report 